\title{
Fresh Per Rectal Bleeding in Pediatric Inflammatory Multisystem Syndrome Temporally Associated with SARS-CoV-2 (PIMS-TS)
}

\author{
Sagar S. Lad ${ }^{1,2}$ (1) Pradeep B. Suryawanshi ${ }^{1} \cdot$ Pranav Jadhav $^{1} \cdot$ Santosh Pandurang Kait ${ }^{1} \cdot$ Preeti Lad $^{1} \cdot$ Jalil Mujawar $^{1}$. \\ Ravindra Khetre ${ }^{1} \cdot$ Prateek Kataria $^{1} \cdot$ Prasad Balte $^{1} \cdot$ Abhinav Neela $^{1} \cdot$ Vishwadeep Kesarwani $^{1} \cdot$ Chandana Bobba $^{1}$
}

Received: 27 January 2021 / Accepted: 5 March 2021 / Published online: 21 March 2021

(C) Dr. K C Chaudhuri Foundation 2021

To the Editor: Gastrointestinal manifestations in PIMS-TS are acute abdomen, diarrhea, and malena. However, there are no reports of per rectal bleeding in these children. A 4-mo-old female infant presented with fever, vomiting, lethargy and fresh blood in stool since 1 day. There was no abdominal distension but bowel sounds were sluggish. She was in compensated shock and needed fluid resuscitation, and respiratory and ionotropic support.

Her investigations showed neutrophilic leucocytosis, raised inflammatory and cardiac markers, and sterile blood culture. Coronavirus disease 2019 (COVID-19) immunoglobulin G ( $\operatorname{IgG}$ ) antibodies were positive $6.26 \mathrm{~S} / \mathrm{CO}(<1.4)$ and reverse transcription polymerase chain reaction (RT-PCR) was negative. The father was COVID-19 RT-PCR positive one month back. She had 3 episodes of fresh blood in stool; hemoglobin dropped to $8 \mathrm{~g} / \mathrm{dL}$; needed transfusion. Echocardiography showed dilated both coronaries with high $\mathrm{z}$ score $>2.5$ Computed tomography showed dilated jejunum and proximal ileum suggestive of obstruction. Exploratory laparotomy showed extensively congested ileum and tiny fibrous band about $2.5 \mathrm{~cm}$ crossing from antimesenteric border to mesentery in proximal ileum. No obvious volvulus was seen. This tiny band cannot cause ischemic changes unless accompanied by volvulus. She was treated with piperacillin-tazobactam, amikacin, immunoglobulin, and methylprednisolone following which inflammatory markers decreased and she was subsequently discharged. On follow-up, she had good weight gain and normal coronaries.

Sagar S. Lad

drsagarlad@yahoo.com

1 Pediatric Intensive Care Unit, Sahyadri Super Speciality Hospital, Shastrinagar, Pune, Maharashtra 411006, India

2 Pediatric Intensive Care Unit, Jehangir Apollo Hospital, Pune, Maharashtra, India
Various studies show that $60 \%$ to $92 \%$ PIMS-TS children have gastrointestinal symptoms [1-3]. The differential diagnosis included hemorrhagic disease of the newborn, Meckel diverticulum, and polyps which were ruled out. Sahn et al. have shown that in PIMS, an inflammatory vascular damage occurs causing bowel thickening and luminal narrowing leading to bowel obstruction. Enterocytes have greatest density of angiotensin-converting enzyme 2 receptors. It is unknown if the inflammatory disease in these areas are due to direct viral induced cellular damage or is the end organ damage of a systemic inflammatory process [4]. Hence, in the current scenario pediatricians should be aware of such unusual manifestations of PIMS-TS.

\section{Declarations}

Conflict of Interest None.

\section{References}

1. WHO. Multisystem inflammatory syndrome in children and adolescents with COVID-19. 2020;(May):1-3.

2. Riphagen S, Gomez X, Gonzalez-Martinez C, Wilkinson N, Theocharis P. Hyperinflammatory shock in children during COVID-19 pandemic. Lancet. 2020;395(10237):1607-8.

3. Verdoni L, Mazza A, Gervasoni A, et al. An outbreak of severe Kawasaki-like disease at the Italian epicentre of the SARS-CoV-2 epidemic: an observational cohort study. Lancet. 2020;395(10239): 1771-8.

4. Sahn B, Eze OP, Edelman MC, et al. Features of intestinal disease associated with COVID-related multisystem inflammatory syndrome in children. J Pediatr Gastroenterol Nutr. 2021;72(3):384-7.

Publisher's Note Springer Nature remains neutral with regard to jurisdictional claims in published maps and institutional affiliations. 